\title{
Development of 3D Image Measurement System for Large Area
}

\author{
[ Yeon Taek OH ]
}

\begin{abstract}
The requirement of large-area and high-precision measurements has increased in industry area. The high-precision multi probe measurement system has been developed to measure the 3D shape. The three different multi probes are combined in measurement system. This system is synchronized between the probes to measure the same position in the sample. Also this paper shows the measurement results with multi probe measurement system
\end{abstract}

Keywords - 3D Image, Synchronization, Multi Probe

\section{Introduction}

Measurement analysis to verify products and parts is critical for efficient industrial research and development. As parts are becoming more precise and greater in size rapidly, the display industry requires image measurement for the related parts.

3D image measurement technology is emerging in the industry that manufactures micro shape. Confocal and white light microscope is used that is an optical measurement technology that does not result in change of the specimen.

Using the current optical measurement technology is also limited to measure a large shape and its height. In particular, there is more and more large-size lens, structures with high aspect ratio and parts that require $20 \mathrm{~mm}$ lateral resolution measurement range because of the rising demand for IT electronic goods and demand to measure the parts is also rising accordingly ${ }^{1)}$. Based on the trend, the study developed a multi probe measurement system that can measure precise images having the same position for specific measurement target by combining multi-purpose sub-micro non-contact probes and sensors that can measure and evaluate large highprecision image, structures with high aspect ratio, and vertical resolution of about $20 \mathrm{~mm}$.

\section{Multi probe Measurement System}

\section{A. System Outline}

Fig. 1 showed the layout of the multi probe measurement system. On the pillar of the device was installed the chromatic confocal and Confocal Laser Scanning Microscope (CLSM).

To set the measuring height of the target object, the probe was placed at the $\mathrm{z}$ stage. The $\mathrm{x}$ and $\mathrm{y}$ stage on the bottom was used to move the object and aligned it with the probe.

Yeon Taek OH

Department of Robot System Engineering, Tongmyong University, KOREA

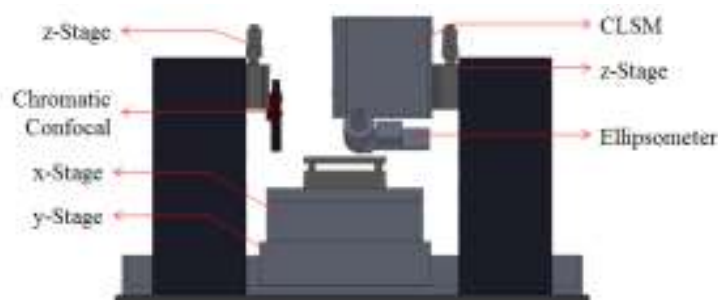

Figure. 1 Layout of 3D measurement system

Fig. 1 showed the layout of the multi probe measurement system. On the pillar of the device was installed the chromatic confocal and Confocal Laser Scanning Microscope (CLSM). To set the measuring height of the target object, the probe was placed at the $\mathrm{z}$ stage. The $\mathrm{x}$ and $\mathrm{y}$ stage on the bottom was used to move the object and aligned it with the probe.

\section{B. Chromatic Confocal}

The chromatic confocal that is a point sensor measured height by scanning the specimen horizontally. It consisted of a head that sends reflected light to the spectrometer from the specimen and controller that analyzes the reflected light. Lateral resolution was decided by the spot size and $\mathrm{z}$ axis resolution was decided by the number of pixels and bits of spectrometer line CCD that detected wave of light reflected from the specimen $^{2)}$. Table 1 showed the specification of chromatic confocal.

TABLE 1 Specification of chromatic confocal

\begin{tabular}{|l|c|}
\hline Measurement Range(Z Axis) & $1100 \mu \mathrm{m}$ \\
\hline Focal Length & $12.7 \mathrm{~mm}$ \\
\hline Measurement Angle & $\pm 27^{\circ}$ \\
\hline Spot Diameter & $4 \mu \mathrm{m}$ \\
\hline XY Resolution & $2 \mu \mathrm{m}$ \\
\hline & \\
\hline
\end{tabular}

Figure 2 Concept chromatic confocal

\section{Confocal Laser scanning microscope}

CLSM using laser as its light source emitted light of a certain range of wavelength from the specimen, passed through the objective lens, separated the exactly focused light 
Proc. of The Second Intl. Conf. On Advances in Civil, Structural and Mechanical Engineering - ACSM 2015

Copyright (C) Institute of Research Engineers and Doctors, USA .All rights reserved.

ISBN: 978-1-63248-074-3 doi: 10.15224/ 978-1-63248-074-3-80

only with detector aperture and sent the digitalized signal to the computer with the detector.

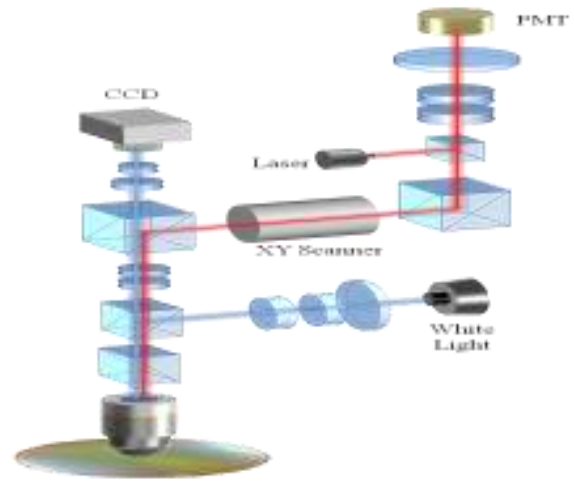

Figure 3 Principle of CLSM $^{3)}$

The field of view of CLSM was decided by the objective lens magnification as shown by Fig. 4. Micro images could be measured in the axial resolution of $10 \mathrm{~nm}$ for every pixel position of lateral resolution. Fig. 4 illustrated the measurement range by the size of objective lens.

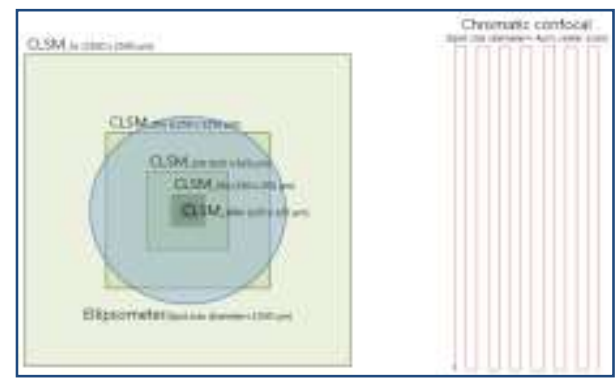

Figure 4 F.O.V.vs Image size(pixels)_CLSM

\section{Ellipsometer}

Ellipsometer is a device that measures film thickness with polarization between incident light and reflective light. The measurement principle was to calculate thickness by matching the conflicting waveform of actually measured reflective light and waveform obtained from simulation.

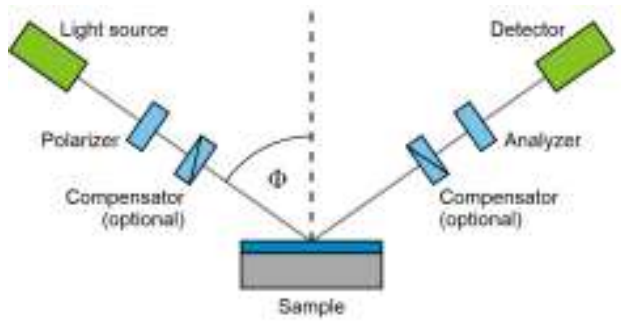

Figure 5 Principle of ellipsometer

The thin film thickness was measured with the actual reflected conflicting waveform obtained from the optical microscope as well as entering reflection coefficient and absorption coefficient depending on the known wavelength to the provided program to change the thickness and match it with the conflicting waveform of measured reflection light. Since it had a bigger beam spot than other measurement probe, the algorithm for convergence that found central position of the beam spot was needed in order to combine the data from other measurement probes.

\section{E. Measurement method analysis using heterogeneous probes}

Different measurement probes had different measurement resolution depending on measuring methods such as lateral resolution, longitudinal resolution and optical spot size. Thus, the result from the specimen measured with each probe in the multi probe system was independent. Though same area was measured ideally like Fig. 6, the measured points were found slightly different from each other. Besides, given that the internal measurement coordinate system of each probe was at different positions and there was error from processing and installation, it was obvious the measured result by each probe was not related with one another.
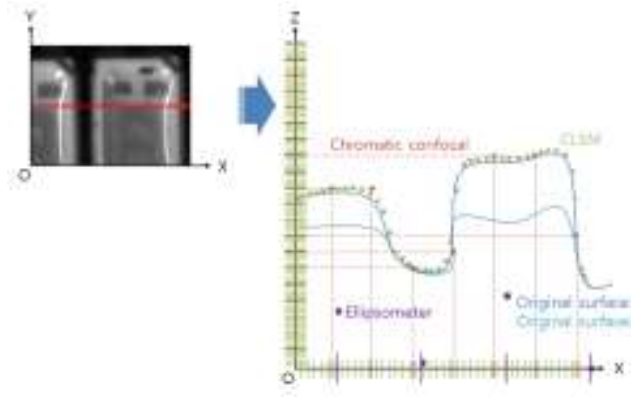

Figure 6 Requirement of synchronization

Even though the same position was measured using different measurement probes, analyzing correlation among the measured data was needed to make the result significant and utilize it. That is, measurement system synchronization technology was needed so that the results that measured form and thickness using chromatic confocal, CLSM and ellipsometer installed at the 3D measurement system were correlated.

\section{Probe Synchronization Control}

Probe synchronization control is aimed to optimize accuracy of the specimen result measured by each probe. The study made chuck for each specimen. It saved the measurement parameter for the specimen measurement location and each probe as recipe and measured the same location of each specimen so that the user could accurately measure the location by using the xy data of the specimen.

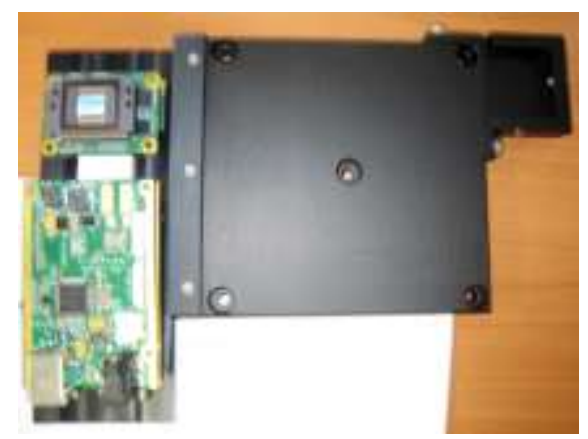

Figure 7 Chuck system for CCD Camera and standard specimen 
Proc. of The Second Intl. Conf. On Advances in Civil, Structural and Mechanical Engineering - ACSM 2015 Copyright ( $)$ Institute of Research Engineers and Doctors, USA .All rights reserved.

ISBN: 978-1-63248-074-3 doi: 10.15224/ 978-1-63248-074-3-80

Actual measurement location and systemic errors of the chromatic confocal were analyzed to measure the same location and off-set was applied as software to improve data accuracy. Besides, CCD camera was used to measure off-set of the chromatic confocal.

The chuck to place specimen on was manufactured like Fig 7 to install the standard specimen and CCD camera4, 5). The $\mathrm{CCD}$ camera can be detached when not in use to measure the spot of the chromatic confocal.

\section{A. XY Stage Mapping}

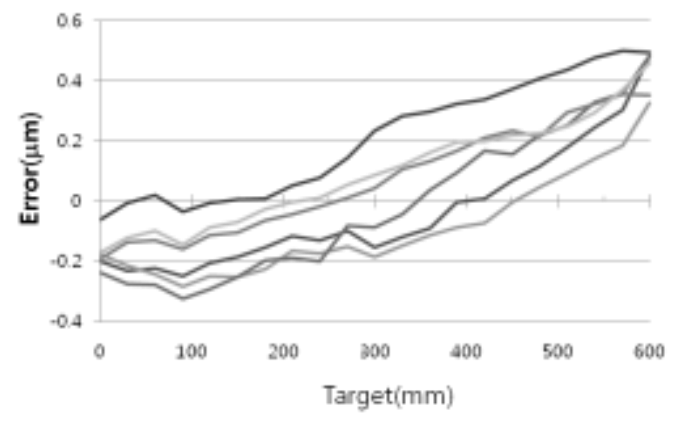

Figure 8 Accuracy test result of y axis

Repeatability was described as $3 \sigma$ of standard deviation. The measurement showed position accuracy of $0.8 \mu \mathrm{m}$ and repeatability of $0.2 \mu \mathrm{m}$.

Error mapping was done using data measured at points with interval of $30 \mathrm{~mm}$ like Fig. 8. The measured value after mapping was listed at Fig. 9. As a result, Fig. 8 had position accuracy of y axis at $0.8 \mu \mathrm{m}$ and Fig. 9 marked $0.2 \mu \mathrm{m}$ after error mapping at the measurement points.

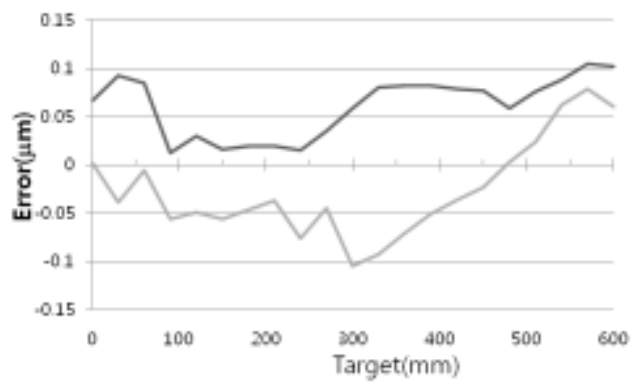

Figure 9 Accuracy test result of y axis after compensation

\section{B. Probe Position Set Up}

At Fig. 10, each probe was installed at the precision stage. Ellipsometer and CLSM were designed to measure the same position of the specimen based on the measuring method and systemic analysis. To minimize measurement time, the moving distance of the specimen was minimized by making it face the chromatic confocal. Through the precise XY stage compensated after mapping, each probe position was identified so that the same position of the specimen could be measured with each probe.

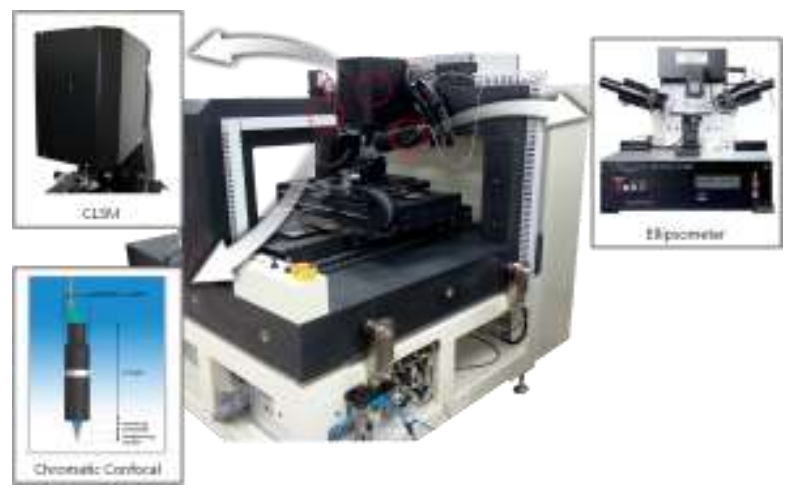

Fig. 10 Multi probe layout of measurement system

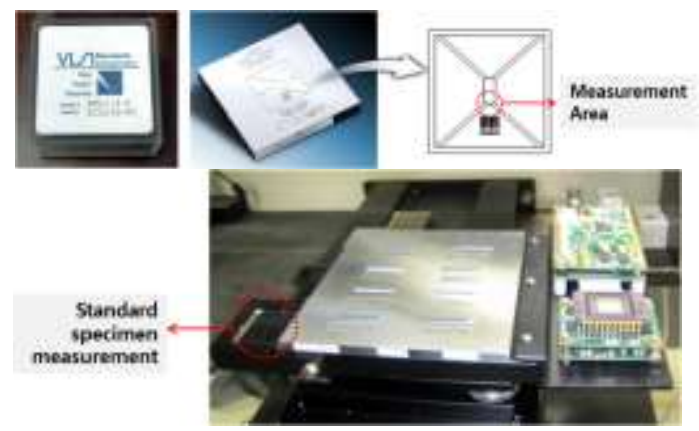

Figure 11 Standard specimen (SHS-1.8Q)

Fig. 11 showed the standard specimen on the chuck. To identify the position of each probe, standard specimen was measured with each probe, the stage encoder value and the measurement data for the standard specimen were analyzed. To see the measurement position of the ellipsometer using the standard specimen, the volume of light reflected from the specimen was measured. The study used reflection volume that changed when the specimen surface was even and its height changed accordingly with regular reflection like Fig. 12. It was found that the reflection changed from 705 to 560 . Besides, as shown by Fig. 13, the height of standard specimen at $1.8 \mu \mathrm{m}$ was measured $1.75 \sim 1.80 \mu \mathrm{m}$ with the chromatic Confocal.
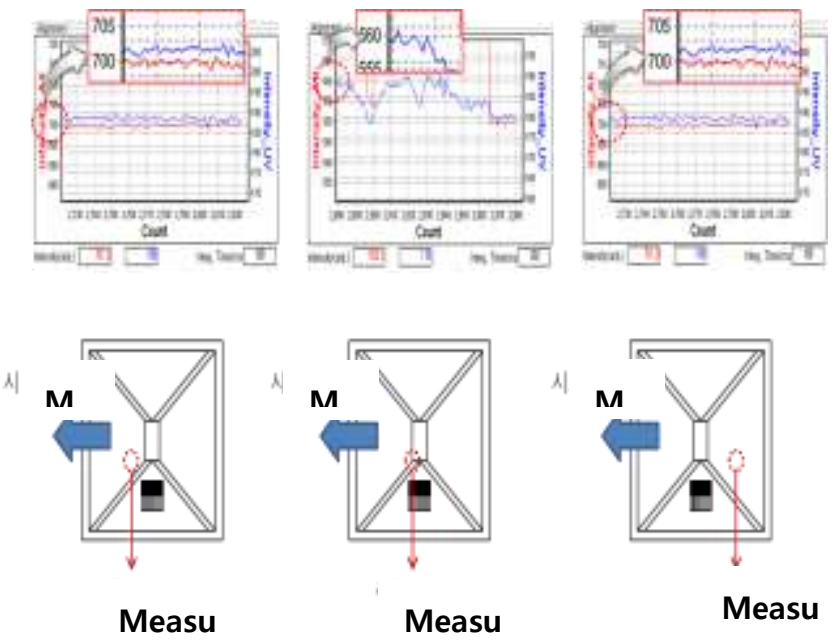

Measu

Measu

Measu 
Proc. of The Second Intl. Conf. On Advances in Civil, Structural and Mechanical Engineering - ACSM 2015

Copyright $@$ Institute of Research Engineers and Doctors, USA .All rights reserved.

ISBN: 978-1-63248-074-3 doi: 10.15224/ 978-1-63248-074-3-80

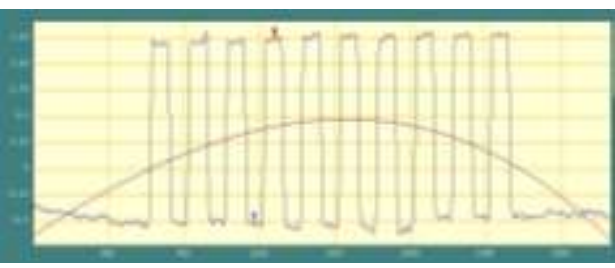

Figure 13 Test result of SHS-1.8Q-chromatic confocal

When the home of the XY stage was assumed $(0,0)$, measuring specific pattern of the standard specimen showed the probes' location like table 2. As CCD camera at CLSM had CCD review function that could show the specimen measurement position, the position of ellipsometer and chromatic confocal with CLSM at the center was shown at table 3 . The measured position data were applied to the upper measurement program.

TABLE 2 Probe position on XY stage

\begin{tabular}{|l|c|c|}
\hline Probe & $\mathrm{x}[\mathrm{mm}]$ & $\mathrm{y}[\mathrm{mm}]$ \\
\hline CLSM & 137.963 & 317.781 \\
\hline Ellipsometer & 134.023 & 310.726 \\
\hline Chromatic confocal & 140.320 & 177.140 \\
\hline
\end{tabular}

TABLE 3 Probe position on CLSM

\begin{tabular}{|l|c|c|}
\hline Probe & $\mathrm{x}[\mathrm{mm}]$ & $\mathrm{y}[\mathrm{mm}]$ \\
\hline CLSM & 0 & 0 \\
\hline Ellipsometer & 3.940 & 7.055 \\
\hline Chromatic confocal & -2.357 & 140.641 \\
\hline
\end{tabular}

If the specimen was moved to CLSM and Chromatic Confocal was selected using CCD function to make the measurement target position as the base, the target position to measure the specimen moved to the Chromatic Confocal position. If the ellipsometer was selected, the position moved to the ellipsometer and the same position of the specimen could be measured with each probe.

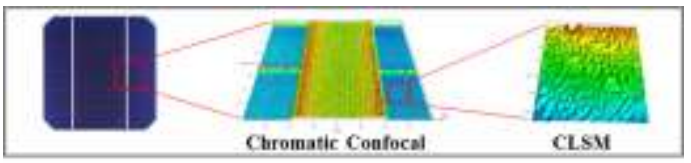

Figure 14 Measurement result using multi probe

Fig. 14 presented the result measured with the multi probe measurement system. With the scanning method for each probe and measurement area considered, the target was quickly measured with the chromatic confocal that can measure profile fast and CLSM was applied to precisely measure the area of interest followed by the data result.

\section{Conclusion}

The existing measurement system measured the position after finding the area assumed to be the same position using the standard like reference point. Doing this required additional devices like precision stage and vision camera for each measuring device. Therefore, measuring the same position using separated different measuring device came with lots of restrictions and difficulty.
However, multi probe-based 3D image measurement system clearly knows the relation between one standard coordinate system and another measurement coordinate system (measurement probe), allowing moving the specimen to the precision stage for measurement more easily. The study suggested arrangement to combine Chromatic confocal, CLSM and ellipsometer considering the principle and condition of each probe to measure. It also suggested how to configure the system to measure large-scale 3D image and how to measure it. Stitching algorithm needs to be developed in the future that can combine $\mathrm{XY}$ stage to expand $\mathrm{x}$ axis measurement area, synchronization of the probe attached on $\mathrm{z}$ axis and the data measured by the probe.

\section{Acknowledgment}

This work was supported by the Technology Innovation Program (or State-of-the Art research Equipment Innovation Program 10038752) funded the Ministry of Trade, Industry \& Energy (MOTIE, KOREA)

\section{References}

[1] Dong-Hyeon Lee \& Nahm-Gyoo Cho, " Assesment of surface profile data acquired by stylus profilometer", Measurement science and technology Vol.23 No.10, 2012

[2] Chih-Kung Lee and Shui-Shong Lu, "Micro-system displacement and profile measurement by an integrated photon tunneling and confocal microscope," The chinese journal of mechanics-series A, Vol.18, No.4, 2002

[3] Takashi KODAMA, Hiroyuki OHTANI, Hideo ARAKAWA and Atsushi IKAI, : Development of confocal laser scanning microscope/atomic force microscope system for force curve measurement", Japanese journal of applied physics, Vol. 43, No. 7B, 2004

[4] Jung Hyun Kim, "Surface roughness measurement of bearing balls using Optical Microscope with Vision Sensor", Jou. of Korean Soc. of Mechanical Technology, Vol. 15(2) pp.149 153, 2013

[5] J. H. Kim, "Design of Piezo driven Motion stage using novel cross hinge structure", Jou. of Korean Soc. of Mechanical Technology, Vol. 14(1) pp.1 5, 2012

About Author:

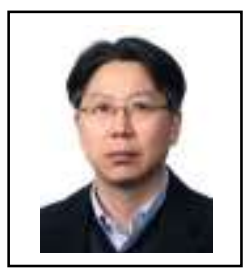

Yeon Taek $\mathrm{OH}$ is a professor in robot system engineering, Tongmyong University. His research interests include metrology, precision Engineering \& robotics. 